\title{
SPACE, TIME, AND MATTER: COSMOLOGICAL PARAMETERS 2001
}

\author{
LAWRENCE M. KRAUSS \\ Departments of Physics and Astronomy \\ Case Western Reserve University, 10900 Euclid Ave. Cleveland, OH 44106-7079
}

\begin{abstract}
Over the past three years, our confidence in the inferred values of cosmological parameters has increased dramatically, confirming that the flat matter dominated Universe that dominated cosmological model building for the past 20 years does not correspond to the Universe in which we live. I review recent developments here and quote best fit current values for fundamental cosmological parameters.

(Invited Review Lecture: Third International Conference on the Identification of Dark Matter, York, England, Sept 2000)
\end{abstract}

\section{Introduction:}

The last decade has witnessed several remarkable transformations in our knowledge of the current state of our universe, including the value of fundamental cosmological parameters. The good news is that the actual values we seem to be converging on are ridiculous. Thus, our increased empirical knowledge has gone hand in hand with increased theoretical confusion. For theorists and observers alike, nothing could be more exciting.

In order to make some attempt to have this review appear less boring than it actually is, I have decided to group the cosmological parameters in terms of three general themes, harking back to the famous book of Hermann Weyl, the title of which nicely encompasses the business of modern cosmology.

\section{Space, The Final Frontier:}

\subsection{Expansion:}

Probably the most important characteristic of the space in which we live is that it is expanding. The expansion rate, given by the Hubble Constant, sets the overall scale for most other observables in cosmology. Thus it is of vital importance to pin down its value if we hope to seriously constrain other cosmological parameters..

Fortunately, over the past five years tremendous strides have been made in our empirical knowledge of the Hubble constant. I briefly review recent developments and prospects for the future here. 


\section{HST-KEY Project:}

This is the largest scale endeavor carried out over the past decade with a goal of achieving a $10 \%$ absolute uncertainty in the Hubble constant. The goal of the project has been to use Cepheid luminosity distances to 25 different galaxies located within 25 Megaparsecs in order to calibrate a variety of secondary distance indicators, which in turn can be used to determine the distance to far further objects of known redshift. This in principle allows a measurement of the distance-redshift relation and thus the Hubble constant on scales where local peculiar velocities are insignificant. The four distance indicators so constrained are: (1) the Tully Fisher relation, appropriate for spirals, (2) the Fundamental plane, appropriate for ellipticals, (3) surface brightness fluctuations, and (4) Supernova Type 1a distance measures.

The HST-Key project has recently reported Hubble constant measurements for each of these methods, which I present below 1 . While I shall adopt these as quoted, it is worth pointing out that some critics of this analysis have stressed that this involves utilizing data obtained by other groups, who themselves sometimes report different values of the Hubble constant for the same data sets.

$$
\begin{gathered}
H_{O}^{T F}=71 \pm 4 \pm 7 \\
H_{O}^{F P}=78 \pm 8 \pm 10 \\
H_{O}^{S B F}=69 \pm 4 \pm 6 \\
H_{O}^{S N 1 a}=68 \pm 2 \pm 5 \\
H_{O}^{W A}=71 \pm 6 \mathrm{kms}^{-1} \mathrm{Mpc}^{-1}(1 \sigma)
\end{gathered}
$$

In the weighted average quoted above, the dominant contribution to the $9 \%$ one sigma error comes from an overall uncertainty in the distance to the Large Magellanic Cloud. If the Cepheid Metallicity were shifted within its allowed $4 \%$ uncertainty range, the best fit mean value for the Hubble Constant from the HST-Key project would shift downard to $68 \pm 6$.

\section{S-Z Effect:}

The Sunyaev-Zeldovich effect results from a shift in the spectrum of the Cosmic Microwave Background radiation due to scattering of the radiation by electgrons as the radiation passes through intervening galaxy clusters on the way to our receivers on Earth. Because the electron temperature in Clusters 
exceeds that in the CMB, the radiation is systematically shifted to higher frequencies, producing a deficit in the intensity below some characteristic frequency, and an excess above it. The amplitude of the effect depends upon the Thompson scattering scross section, and the electron density, integrated over the photon's path:

$$
\mathrm{SZ} \approx \int \sigma_{T} n_{e} d l
$$

At the same time the electrons in the hot gas that dominates the baryonic matter in galaxy clusters also emits X-Rays, and the overall X-Ray intensity is proportional to the square of the electron density integrated along the line of sight through the cluster:

$$
\mathrm{X}-\mathrm{Ray} \approx \int n_{e}^{2} d l
$$

Using models of the cluster density profile one can then use the the differing dependence on $n_{e}$ in the two integrals above to extract the physical path-length through the cluster. Assuming the radial extension of the cluster is approximately equal to the extension across the line of sight one can compare the physical size of the cluster to the angular size to determine its distance. Clearly, since this assumption is only good in a statistical sense, the use of S-Z and X-Ray observations to determine the Hubble constant cannot be done reliably on the basis of a single cluster observation, but rather on an ensemble.

A recent preliminary analysis of several clusters 1 yields:

$$
H_{0}^{S Z}=60 \pm 10 k s^{-1} M p c^{-1}
$$

Type 1a SN (non-Key Project):

One of the HST Key Project distance estimators involves the use of Type 1a SN as standard candles. As previously emphasized, the Key Project does not perform direct measurements of Type 1a supernovae but rather uses data obtained by other gorpus. When these groups perform an independent analysis to derive a value for the Hubble constant they arrive at a smaller value than that quoted by the Key Project. Their most recent quoted value is $\mathrm{B}$ :

$$
H_{0}^{1 a}=64_{-6}^{+8} k s^{-1} M p c^{-1}
$$


At the same time, Sandage and collaborators have performed an independent analysis of SNe Ia distances and obtain 4 :

$$
H_{0}^{1 a}=58 \pm 6 k s^{-1} M p c^{-1}
$$

\section{Surface Brightness Fluctuations and The Galaxy Density Field:}

Another recently used distance estimator involves the measurement of fluctuations in the galaxy surface brightness, which correspond to density fluctuations allowing an estimate of the physical size of a galaxy. This measure yields a slightly higher value for the Hubble constant 0 :

$$
H_{0}^{S B F}=74 \pm 4 k s^{-1} M p c^{-1}
$$

Time Delays in Gravitational Lensing:

One of the most remarkable observations associated with observations of multiple images of distant quasars due to gravitational lensing intervening galaxies has been the measurement of the time delay in the two images of quasar $Q 0957+561$. This time delay, measured quite accurately to be $417 \pm 3$ days is due to two factors: The path-length difference between the quasar and the earth for the light from the two different images, and the Shapiro gravitational time delay for the light rays traveling in slightly different gravitational potential wells. If it were not for this second factor, a measurement of the time delay could be directly used to determine the distance of the intervening galaxy. This latter factor however, implies that a model of both the galaxy, and the cluster in which it is embedded must be used to estimate the Shapiro time delay. This introduces an additional model-dependent uncertainty into the analysis. Two different analyses yield values 6 :

$$
\begin{aligned}
& H_{0}^{T D 1}=69_{-12}^{+18}(1-\kappa) k s^{-1} M p c^{-1} \\
& H_{0}^{T D 2}=74_{-10}^{+18}(1-\kappa) k s^{-1} M p c^{-1}
\end{aligned}
$$

where $\kappa$ is a parameter which accounts for a possible deviation in cluster parameters governing the overall induced gravitational time delay of the two signals from that assumed in the best fit. It is assumed in the analysis that $\kappa$ is small. 


\section{Summary:}

It is difficult to know how to best incorporate all of the quoted estimates into a single estimate, given their separate systematic and statistical uncertainties. Assuming large number statistics, where large here includes the nine quoted values, I perform a simple weighted average of the individual estimates, and find an approximate average value:

$$
H_{0}^{A v} \approx 68 \pm 3 k s^{-1} M p c^{-1}
$$

\subsection{Geometry:}

It has remained a dream of observational cosmologists to be able to directly measure the geometry of space-time rather than infer the curvature of the universe by comparing the expansion rate to the mean mass density. While several such tests, based on measuring galaxy counts as a function of redshift, or the variation of angular diameter distance with redshift, have been attempted in the past, these have all been stymied by the achilles heel of many observational measurements in cosmology, evolutionary effects.

Recently, however, measurements of the cosmic microwave background have finally brought us to the threshold of a direct measurement of geometry, independent of traditional astrophysical uncertainties. The idea behind this measurement is, in principle, quite simple. The CMB originates from a spherical shell located at the surface of last scattering (SLS), at a redshift of roughly $z \approx 1000$ ):

If a fiducial length could unambigously be distinguished on this surface, then a determination of the angular size associated with this length would allow a determination of the intervening geometry:

Fortunately, nature has provided such a fiducial length, which corresponds roughly to the horizon size at the time the surface of last scattering existed. The reason for this is also straightforward. This is the largest scale over which causal effects at the time of the creation of the surface of last scattering could have left an imprint. Density fluctuations on such scales would result in acoustic oscillations of the matter-radiation fluid, and the doppler motion of electrons moving along with this fluid which scatter on photons emerging from the SLS produces a characteristic peak in the power spectrum of fluctuations of the CMBR at a wavenumber corresponding to the angular scale spanned by this physical scale. These fluctuations should also be visually distinguishable in an image map of the $\mathrm{CMB}$, provided a resolution on degree scales is possible.

Recently, two different ground-based balloon experiments, one launched in Texas and one launched in Antarctica have resulted in maps with the re- 
COSMIC MICROWAVE BACKGROUND

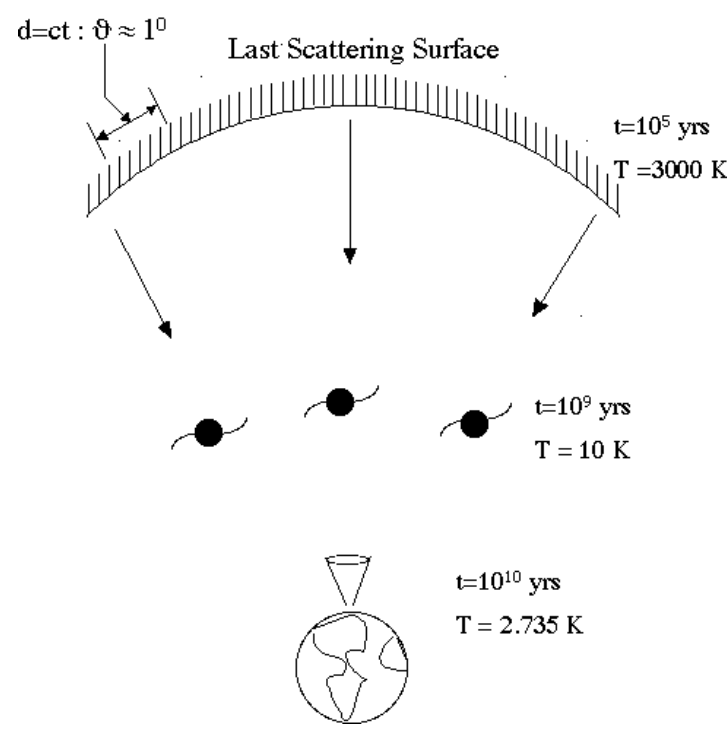

Figure 1. A schematic diagram of the surface of last scattering, showing the distance traversed by CMB radiation.

quired resolution $\mathrm{A}$. $\mathrm{A}$. Shown below is a comparison of the actual boomerang map with several simulations based on a gaussian random spectrum of density fluctuations in a cold-dark matter universe, for open, closed, and flat cosmologies. Even at this qualitative level, it is clear that a flat universe provides better agreement to between the simulations and the data than either an open or closed universe?

On a more quantitative level, one can compare the inferred power spectra with predicted spectra 6 . Such comparisions, for both the Boomerang and Maxima results yields a constraint on the density parameter: 


\section{Angular Size of a Fixed Scale in Open, Closed, and Flat Universes:}

First Scale to Collapse after Recombination $(\approx$ distance spanned by light ray =horizon size)

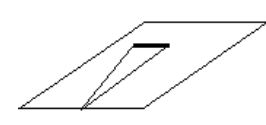

flat
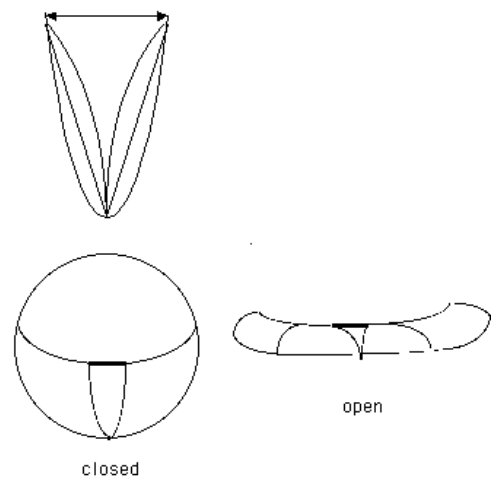

open

Figure 2. The geometry of the Universe and ray trajectories for CMB radiation.

$$
\Omega=1.1 \pm .12(95 \% C L)
$$

For the first time, it appears that the longstanding prejudice of theorists, namely that we live in a flat universe, may have been vindicated by observation! However, theorists can not be too self-satisfied by this result, because the source of this energy density appears to be completely unexpected, and largely inexplicable at the present time, as we will shortly see.

\section{Time}



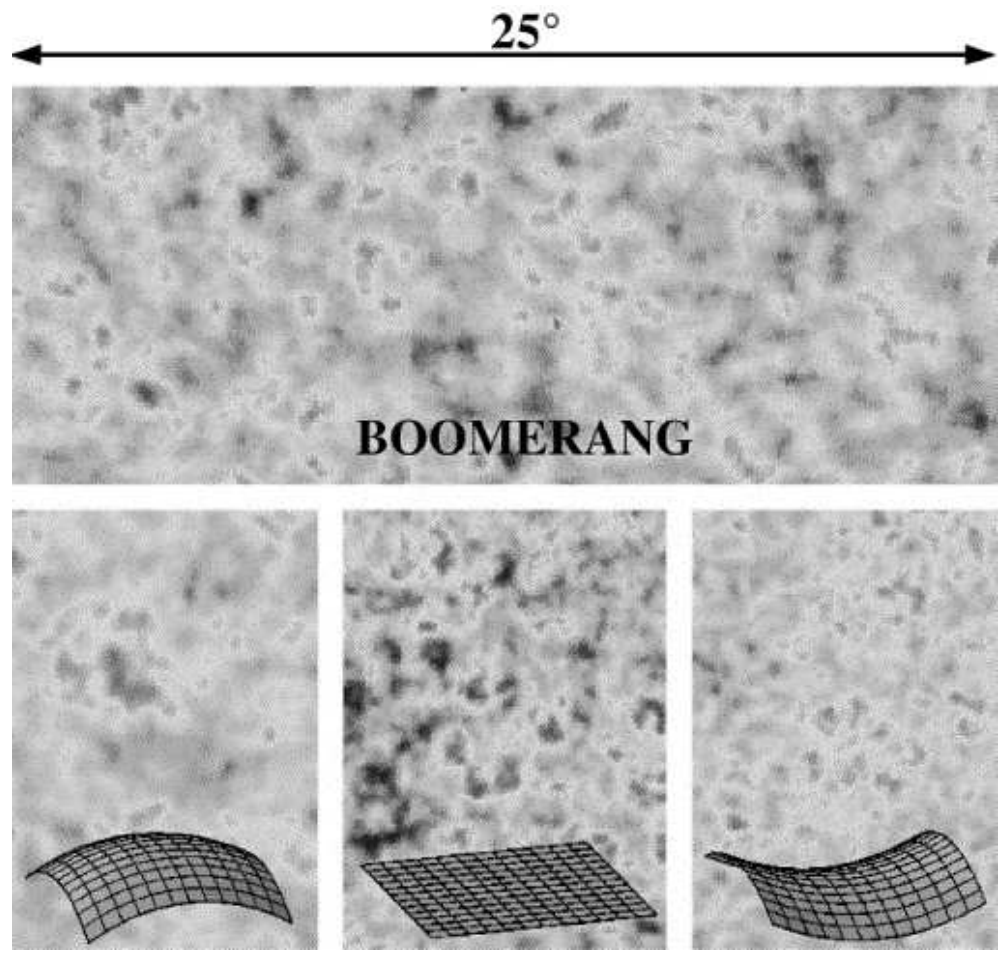

Figure 3. Boomerang data visually compared to expectations for an open, closed, and flat CDM Universe.

\subsection{Stellar Ages:}

Ever since Kelvin and Helmholtz first estimated the age of the Sun to be less than 100 million years, assuming that gravitational contraction was its prime energy source, there has been a tension between stellar age estimates and estimates of the age of the universe. In the case of the Kelvin-Helmholtz case, the age of the sun appeared too short to accomodate an Earth which was several billion years old. Over much of the latter half of the 20th century, the opposite problem dominated the cosmological landscape. Stellar ages, based on nuclear reactions as measured in the laboratory, appeared to be too old to accomodate even an open universe, based on estimates of the Hubble parameter. Again, as I shall outline in the next section, the observed expansion rate gives an upper limit on the age of the Universe which depends upon the 
equation of state, and the overall energy density of the dominant matter in the Universe.

There are several methods to attempt to determine stellar ages, but I will concentrate here on main sequence fitting techiniques, because those are the ones I have been involved in.

The basic idea behind main sequence fitting is simple. A stellar model is constructed by solving the basic equations of stellar structure, including conservation of mass and energy and the assumption of hydrostatic equilibrium, and the equations of energy transport. Boundary conditions at the center of the star and at the surface are then used, and combined with assumed equation of state equations, opacities, and nuclear reaction rates in order to evolve a star of given mass, and elemental composition.

Globular clusters are compact stellar systems containing up to $10^{5}$ stars, with low heavy element abundance. Many are located in a spherical halo around the galactic center, suggesting they formed early in the history of our galaxy. By making a cut on those clusters with large halo velocities, and lowest metallicities (less than 1/100th the solar value), one attempts to observationally distinguish the oldest such systems. Because these systems are compact, one can safely assume that all the stars within them formed at approximately the same time.

Observers measure the color and luminosity of stars in such clusters, producing colpr-magnitude diagrams of the type shown in Figure 2 (based on data from 10 .

Next, using stellar models, one can attempt to evolve stars of differing mass for the metallicities appropriate to a given cluster, in order to fit observations. A point which is often conveniently chosen is the so-called main sequence-turnoff (MSTO) point, the point in which hydrogen burning (main sequence) stars have exhausted their supply of hydrogen in the core. After the MSTO, the stars quickly expand, become brighter, and are referred to as Red Giant Branch (RGB) stars. Higher mass stars develop a helium core that is so hot and dense that helium fusion begins. These form along the horizontal branch. Some stars along this branch are unstable to radial pulsations, the so-called RR Lyrae stars mentioned earlier, which are important distance indicators. While one in principle could attempt to fit theoretical isochrones (the locus of points on the predicted CM curve corresponding to different mass stars which have evolved to a specified age), to observations at any point, the main sequence turnoff is both sensitive to age, and involves minimal (though just how minimal remains to be seen) theoretical uncertainties.

Dimensional analysis tells us that the main sequence turnoff should be a sensitive function of age. The luminosity of main sequence stars is very

idmreview: submitted to World Scientific on October 31, 2018 


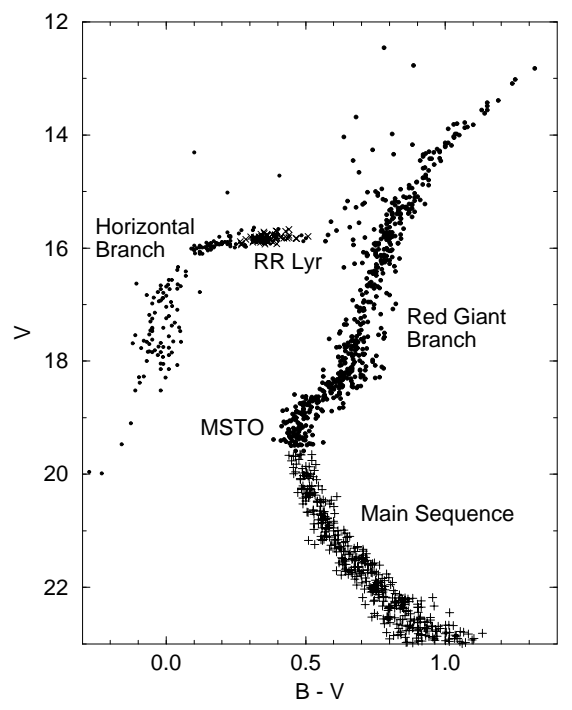

Figure 4. Color-magnitude diagram for a typical globular cluster, M15. Vertical axis plots the magnitude (luminosity) of the stars in the $\mathrm{V}$ wavelength region and the horizontal axis plots the color (surface temperature) of the stars.

roughly proportional to the third power of solar mass. Hence the time it takes to burn the hydrogen fuel is proportional to the total amount of fuel (proportional to the mass $\mathrm{M}$ ), divided by the Luminosity - proportional to $M^{3}$. Hence the lifetime of stars on the main sequence is roughly proportional to the inverse square of the stellar mass.

Of course the ability to go beyond this rough approximation depends completely on the on the confidence one has in one's stellar models. It is worth noting that several improvements in stellar modeling have recently combined to lower the overall age estimates of globular clusters. The inclusion of diffusion lowers the age of globular clusters by about $7 \%$ 11 , and a recently improved equation of state which incorporates the effect of Coulomb interactions 12 has lead to a further $7 \%$ reduction in overall ages. Of course, what is most important for the comparison of cosmological predictions with inferred age estimates is the uncertainties in these and other stellar model parameters, and not merely their best fit values.

Over the course of the past several years, I and my collaborators have tried to incorporate stellar model uncertainties, along with observational uncertainties into a self consistent Monte Carlo analysis which might allow one 
to estimate a reliable range of globular cluster ages. Others have carried out independent, but similar studies, and at the present time, rough agreement has been obtained between the different groups (i.e. see13).

I will not belabor the detailed history of all such efforts here. The most crucial insight has been that stellar model uncertainties are small in comparison to an overall observational uncertainty inherent in fitting predicted main sequence luminosities to observed turnoff magnitudes. This matching depends crucially on a determination of the distance to globular clusters. The uncertainty in this distance scale produces by far the largest uncertainty in the quoted age estimates.

In many studies, the distance to globular clusters can be parametrized in terms of the inferred magnitude of the horizontal branch stars. This magnitude can, in turn, be presented in terms of the inferred absolute magnitude, $M_{v}(\mathrm{RR})$ of RR Lyrae variable stars located on the horizontal branch.

In 1997, the Hipparcos satellite produced its catalogue of parallaxes of nearby stars, causing an apparent revision in distance estimates. The Hipparcos parallaxes seemed to be systematically smaller, for the smallest measured parallaxes, than previous terrestrially determined parallaxes. Could this represent the unanticipated systematic uncertainty that David has suspected? Since all the detailed analyses had been pre-Hipparcos, several groups scrambled to incorporate the Hipparcos catalogue into their analyses. The immediate result was a generally lower mean age estimate, reducing the mean value to 11.5-12 Gyr, and allowing ages of the oldest globular clusters as low as 9.5 Gyr. However, what is also clear is that there is now an explicit systematic uncertainty in the RR Lyrae distance modulus which dominates the results. Different measurements are no longer consistent. Depending upon which distance estimator is correct, and there is now better evidence that the distance estimators which disagree with Hipparcos-based main sequence fitting should not be dismissed out of hand, the best-fit globular cluster estimate could shift up perhaps $1 \sigma$, or about $1.5 \mathrm{Gyr}$, to about $13 \mathrm{Gyr}$.

Within the past two years, Brian Chaboyer and I have reanalyzed globular cluster ages, incorporating new nuclear reaction rates, cosmological estimates of the ${ }^{4} \mathrm{He}$ abundance, and most importantly, several new estimates of $M_{v}(\mathrm{RR})$. The result is that while systematic uncertainties clearly still dominate, we argue that the mean age of the oldest globular clusters has increased about 1 Gyr, to be $12.7_{-2}^{+3}(95 \%)$ Gyr, with a $95 \%$ confidence range of about 11-16 Gyr ?. It is this range that I shall now compare to that determined using the Hubble estimates given earlier. 


\subsection{Hubble Age:}

As alluded to earlier, in a Friedman-Robertson-Walker Universe, the age of the Universe is directly related to both the overall density of energy, and to the equation of state of the dominant component of this energy density. The equation of state is parameterized by the ratio $\omega=p / \rho$, where $p$ stands for pressure and $\rho$ for energy density. It is this ratio which enters into the second order Friedman equation describing the change in Hubble parameter with time, which in turn determines the age of the Universe for a specific net total energy density.

The fact that this depends on two independent parameters has meant that one could reconcile possible conflicts with globular cluster age estimates by altering either the energy density, or the equation of state. An open universe, for example, is older for a given Hubble Constant, than is a flat universe, while a flat universe dominated by a cosmological constant can be older than an open matter dominated universe.

If, however, we incorporate the recent geometric determination which suggests we live in a flat Universe into our analysis, then our constraints on the possible equation of state on the dominant energy density of the universe become more severe. If, for existence, we allow for a diffuse component to the total energy density with the equation of state of a cosmological constant $(\omega=-1)$, then the age of the Universe for various combinations of matter and cosmological constant are shown below.

Table 1. Hubble Ages for a Flat Universe, $H_{0}=68 \pm 6$, (" $\left.2 \sigma "\right)$

\begin{tabular}{|l|l|c|}
\hline$\Omega_{M}$ & $\Omega_{x}$ & $t_{0}$ \\
\hline 1 & 0 & $9.7 \pm 1$ \\
0.2 & 0.8 & $15.3 \pm 1.5$ \\
0.3 & 0.7 & $13.7 \pm 1.4$ \\
0.35 & 0.65 & $12.9 \pm 1.3$ \\
\hline
\end{tabular}

Clearly, a matter-dominated flat universe is in trouble if one wants to reconcile the inferred Hubble age with the lower limit on the age of the universe inferred from globular clusters. In fact, if one took the above constraints at face value, such a Universe is ruled out on the basis of age estimates and the Hubble constant estimates. However, I am old enough to know that systematic uncertainties in cosmology often shift parameters well outside their formal two sigma, or even three sigma limits. In order to definitely rule out a flat matter dominated universe using a comparison of stellar and Hubble ages,

idmreview: submitted to World Scientific on October 31, 2018 
uncertainties in both would have to be reduced by at least a factor of two.

\section{Matter}

Having indirectly probed the nature of matter in the Universe using the previous estimates, it is now time to turn to direct constraints that have been derived in the past decade. Here, perhaps more than any other area of observational cosmology, new observations have changed the way we think about the Universe.

\subsection{The Baryon Density: a re-occuring crisis?:}

The success of Big Bang Nucleosynthesis in predicting in the cosmic abundances of the light elements has been much heralded. Nevertheless, the finer the ability to empirically infer the primordial abundances on the basis of observations, the greater the ability to uncover some small deviation from the predictions. Over the past five years, two different sets of observations have threatened, at least in some people's minds, to overturn the simplest BBN model predictions. I believe it is fair to say that most people have accepted that the first threat was overblown. The concerns about the second have yet to fully subside.

i. Primordial Deuterium: The production of primordial deuterium during $\mathrm{BBN}$ is a monotonically decreasing function of the baryon density simply because the greater this density the more efficiently protons and neutrons get processed to helium, and deuterium, as an intermediary in this reactions set, is thus also more efficiently processed at the same time. The problem with inferring the primordial deuterium abundance by using present day measurements of deuterium abundances in the solar system, for example, is that deuterium is highly processed (i.e. destroyed) in stars, and no one has a good enough model for galactic chemical evolution to work backwards from the observed abundances in order to adequately constrain deuterium at a level where this constraint could significantly test BBN estimates.

Three years ago, the situation regarding deuterium as a probe of BBN changed dramatically, when David Tytler and Scott Burles convincingly measured the deuterium fraction in high redshift hydrogen clouds that absorb light from even higher redshift quasars. Because these clouds are at high redshift, before significant star formation has occurred, little post BBN deuterium processing is thought to have taken place, and thus the measured value gives a reasonable handle on the primordial BBN abundance. The best measured system 14 yields a deuterium to hydrogen fraction of

idmreview: submitted to World Scientific on October 31, 2018 


$$
(D / H)=(3.3 . \pm 0.5) \times 10^{-5}(2 \sigma)
$$

This, in turn, leads to a contraint on the baryon fraction of the Universe, via standard $\mathrm{BBN}$,

$$
\Omega_{B} h^{2}=.0190 \pm .0018 \quad(2 \sigma)
$$

where the quoted uncertainty is dominated by the observational uncertainty in the $\mathrm{D} / \mathrm{H}$ ratio, and where $H_{0}=100 h$. Thus, taken at face value, we now know the baryon density in the universe today to an accuracy of about $10 \%$ !

When first quoted, this result sent shock waves through some of the BBN community, because this value of $\Omega_{B}$ is only consistent if the primordial helium fraction (by mass) is greater than about $24.5 \%$. However, a number of previous studies had claimed an upper limit well below this value. After the dust has settled, it is clear that these previous claims are likely to under-estimated systematic observational effects. Recent studies, for example, place an upper limit on the primordial helium fraction closer to $25 \%$.

In any case, even if somehow the deuterium estimate is wrong, one can combine all the other light element constraints to produce a range for $\Omega_{b} h^{2}$ consistent with observation:

$$
\Omega_{B} h^{2}=.016-0.025
$$

ii. $C M B$ constraints: Beyond the great excitement over the observation of a peak in the CMB power spectrum at an angular scale corresponding to that expected for a flat universe lay some excitement/concern over the small apparent size of the next peak in the spectrum, at higher multipole moment (smaller angular size). The height of the first peak in the CMB spectrum is related to a number of cosmological parameters and thus cannot alone be used to constrain any one of them. However, the relative height of the first and second peaks is strongly dependent on the baryon fraction of the universe, since the peaks themselves arise from compton scattering of photons off of electrons in the process of becoming bound to baryons. Analyses of the two most recent small-scale CMB results produces a claimed constraint 6 :

$$
\Omega_{B} h^{2}=.032 \pm .009 \quad(2 \sigma)
$$


Depending upon how you look at this, this is either a stunning confirmation that the overall scale for $\Omega_{B}$ predicted by simple BBN analyses is correct, or a horrible crisis, in which the two constraints, one from primordial deuterium, and one from CMB observations, disagree at the two sigma level. Given the history of this subject, I expect the former response is perhaps appropriate for the moment. In particular, the CMB results are the very first to probe this regime, and first observations are often suspect, and in addition, the CMB peak heights do have a dependence on other cosmological parameters which must be fixed in order to derive the above constraint on $\Omega_{B}$.

Assuming the Burles and Tytler limit on $\Omega_{B} h^{2}$ is correct, and taking the range for $H_{0}$ given earlier, one derives the constraint on $\Omega_{B}$ of

$$
\Omega_{B}=.045 \pm 0.15
$$

\section{2 $\Omega_{\text {matter }}$}

Perhaps the greatest change in cosmological prejudice in the past decade relates to the inferred total abundance of matter in the Universe. Because of the great intellectual attraction Inflation as a mechanism to solve the so-called Horizon and Flatness problems in the Universe, it is fair to say that most cosmologists, and essentially all particle theorists had implicitly assumed that the Universe is flat, and thus that the density of dark matter around galaxies and clusters of galaxies was sufficient to yield $\Omega=1$. Over the past decade it became more and more difficult to defend this viewpoint against an increasing number of observations that suggested this was not, in fact, the case in the Universe in which we live.

The earliest holes in this picture arose from measurements of galaxy clustering on large scales. The transition from a radiation to matter dominated universe at early times is dependent, of course, on the total abundance of matter. This transition produces a characteristic signature in the spectrum of remnant density fluctuations observed on large scales. Making the assumption that dark matter dominates on large scales, and moreover that the dark matter is cold (i.e. became non-relativistic when the temperature of the Universe was less than about a keV fits to the two point correlation function of galaxies on large scales yielded 15,16 :

$$
\Omega_{M} h=.2-.3
$$


Unless $h$ was absurdly small, this would imply that $\Omega_{M}$ is substantially less than 1 .

The second nail in the coffin arose when observations of the evolution of large scale structure as a function of redshift began to be made. Bahcall and collaborators 17 argued strongly that evidence for any large clusters at high redshift would argue strongly against a flat cold dark matter dominated universe, because in such a universe structure continues to evolve with redshift up to the present time on large scales, so that in order to be consistent with the observed structures at low redshift, far less structure should be observed at high redshift. Claims were made that an upper limit $\Omega_{B} \leq 0.5$ could be obtained by such analyses.

A number of authors have questioned the systematics inherent in the early claims, but it is certainly clear that there appears to be more structure at high redshift than one would naively expect in a flat matter dominated universe. Future studies of X-ray clusters, and use of the Sunyaev-Zeldovich effect to measure cluster properties should be able to yield measurements which will allow a fine-scale distinction not just between models with different overall dark matter densities, but also models with the same overall value of $\Omega$ and different equations of state for the dominant energy 18 .

For the moment, however, perhaps the best overall constraint on the total density of clustered matter in the universe comes from the combination of XRay measurements of clusters with large hydrodynamic simulations. The idea is straightforward. A measurement of both the temperature and luminosity of the X-Rays coming from hot gas which dominates the total baryon fraction in clusters can be inverted, under the assumption of hydrostatic equilibrium of the gas in clusters, to obtain the underlying gravitational potential of these systems. In particular the ratio of baryon to total mass of these systems can be derived. Employing the constraint on the total baryon density of the Universe coming from BBN, and assuming that galaxy clusters provide a good mean estimate of the total clustered mass in the Universe, one can they arrive at an allowed range for the total mass density in the Universe 19.22.21. Many of the initial systematic uncertainties in this analysis having to do with cluster modelling have ngw been dealt with by better observations, and better simulations (i.e. see22), so that now a combination of BBN and cluster measurements yields:

$$
\Omega_{M}=0.35 \pm 0.1 \quad(2 \sigma)
$$




\subsection{Equation of State of Dominant Energy:}

Remarkably, the above estimate for $\Omega_{M}$ brings the discussion of cosmological parameters full circle, with consistency obtained for a flat 12.5 billion year old universe, but not one dominated by matter. As noted previously, a cosmological constant dominated universe with $\Omega_{M}=0.35$ has an age which nicely fits in the best-fit range. However, based on the data discussed thus far, we have no direct evidence that the dark energy necessary to result in a flat universe actually has the equation of state appropriate for a vacuum energy. Direct motivation for the possibility that the dominant energy driving the expansion of the Universe violates the Strong Energy Condition came, in 1998, from two different sets of observations - fdistant Type 1a Supernovae. In measuring the distance-redshift relation 23,24 these groups both came to the same, surprising conclusion: the expansion of the Universe seems to be accelerating! This is only possible if the dominant energy is "cosmologicalconstant-like", namely if " $\omega<-0.5$ (recall that $\omega=-1$ for a cosmological constant).

In order to try and determine if the dominant dark energy does in fact differ significantly from a static vacuum energy — as for example may occur if some background field that is dynamically evolving is dominating the expansion energy at the moment - one can hope to search for deviations from the distance-redshift relation for a cosmological constant-dominated universe. To date, none have been observed. In fact, existing measurements already put an upper limit $\omega \leq-0.6$. Recent work ? suggests that the best one might be able to do from the ground using SN measurements would be to improve this limit to $\omega \leq-0.7$. Either other measurements, such as galaxy cluster evolution observations, or space-based SN observations would be required to further tighten the constraint.

\section{Conclusions: A Cosmic Uncertainty Principle}

I list the overall constraints on cosmological parameters discussed in this review in the table below. It is worth stressing how completely remarkable the present situation is. After 20 years, we now have the first direct evidence that the Universe might be flat, but we also have definitive evidence that there is not enough matter, including dark matter, to make it so. We seem to be forced to accept the possibility that some weird form of dark energy is the dominant stuff in the Universe. It is fair to say that this situation is more mysterious, and thus more exciting, than anyone had a right to expect it to be.

idmreview: submitted to World Scientific on October 31, 2018 
Table 2. Cosmological Parameters 2001

\begin{tabular}{|l|l|c|}
\hline Parameter & Allowed range & Formal Conf. Level (where approp.) \\
\hline$H_{0}$ & $68 \pm 6$ & $2 \sigma$ \\
$t_{0}$ & $12.7_{-2}^{+3}$ & $2 \sigma$ \\
$\Omega_{B} h^{2}$ & $.019 \pm .0018$ or $.032 \pm .009$ & \\
$\Omega_{B}$ & $0.045 \pm 0.015$ & $2 \sigma$ \\
$\Omega_{M}$ & $0.35 \pm 0.1$ & $2 \sigma$ \\
$\Omega_{T O T}$ & $1.1 \pm 0.12$ & $2 \sigma$ \\
$\Omega_{X}$ & $0.65 \pm 0.15$ & $2 \sigma$ \\
$\omega$ & $\leq-0.6$ & $2 \sigma$ \\
\hline
\end{tabular}

The new situation changes everything about the way we think about cosmology. In the first place, it demonstrates that Geometry and Destiny are no longer linked. Previously, the holy grail of cosmology involved determining the density parameter $\Omega$, because this was tantamount to determining the ultimate future of our universe. Now, once we accept the possibility of a non-zero cosmological constant, we must also accept the fact that any universe, open, closed, or flat, can either expand forever, or reverse the present expansion and end in a big crunch 27. But wait, it gets worse, as my colleague Michael Turner and I have also demonstrated, there is no set of cosmological measurements, no matter how precise, that will allow us to determine the ultimate future of the Universe. In order to do so, we would require a theory of everything.

On the other hand, if our universe is in fact dominated by a cosmological constant, the future for life is rather bleak 28 . Distant galaxies will soon blink out of sight, and the Universe will become cold and dark, and uninhabitable....

This bleak picture may seem depressing, but the flip side of all the above is that we live in exciting times now, when mysteries abound. And these mysteries, or new ones, are likely to remain with us for some time.

\section{Acknowledgments}

I thank my collaborators involved in various aspects of my own work described here, including Michael Turner, Brian Chaboyer, Craig Copi, and Glenn Starkman, and also the observers whose results have helped make cosmology so exciting in the past decade. I also thank the the organizers for a very stimulating and enjoyable meeting. 


\section{References}

1. J. Mould et al, Ap. J., 529, 786 (2000)

2. M. Birkinshaw, Phys. Rep. , 310, 97 (1999)

3. J. Saurabh et al, Ap. J. Suppl. 125, 73 (1999)

4. B. R Parodi et al, Ap. J. 540, 634 (2000)

5. J. P. Blakeslee et al, Ap. J. Lett. 527, 73 (1999)

6. K-H. Chae, Ap. J. 524, 582 (1999)

7. P. de Bernardis et al, Nature 404, 995 (2000)

8. S. Hanany et al, Ap. J. Lett. 545, 5 (2000)

9. A. H. Jaffe et al, astro-ph/0007333 (2000)

10. P.R. Durrell and W. E. Harris, AJ, 105, 1420 (1993)

11. B. Chaboyer, P. Demarque, and A. Sarajedini, Ap. J. 459, 558 (1996)

12. B. Chaboyer, and Y.-C. Kim, Ap.J. 454, 76 (1995)

13. L. M. Krauss, Phys Rep.333-334, 33 (2000)

14. S. Burles and D. Tytler, Ap. J. 499, 699 (1998)

15. J. A. Peacock and S. J. Dodds, MNRAS 280, 19 (1996)

16. A. Liddle et al, MNRAS 278, 644 (1996); 282, 281 (1996)

17. N. A. Bahcall et al Ap. J. Lett. 485, 53 (1997)

18. Z. Haiman et al, astro-ph/0002336 (2000)

19. S. D. M. White et al, MNRAS 262, 1023 (1993)

20. L. M. Krauss, Ap. J. 501, 461 (1998)

21. A. E. Evrard, MNRAS 292, 289 (1997)

22. J. Mohr et al astro-ph/0004244 (2000)

23. S. Perlmutter et al, Ap. J. 517, 565 (1999)

24. B. Schmidt et al, Ap. J. 507, 46 (1998)

25. S. Perlmutter, M.S. Turner, M. White, Phys. Rev. Lett. 83, 670 (1999)

26. L.M. Krauss, E. Linton, D. Davis, M. Grugel, to appear.

27. L. M. Krauss and M. S. Turner, J. Gen. Rel. Grav. 31, 1453 (1999)

28. L. M. Krauss and G. Starkmann, Ap. J. 531, 22 (2000) 